\title{
Growing competitive or tolerant? Significance of apical dominance in the overcompensating herb Gentianella campestris
}

\author{
Tommy Lennartsson, ${ }^{1,4}$ Satu Ramula, ${ }^{2}$ and Juha Tuomi ${ }^{2,3}$ \\ ${ }^{1}$ Swedish Biodiversity Centre, Swedish University of Agricultural Sciences, Box 7016, 75007 Uppsala, Sweden \\ ${ }^{2}$ Section of Ecology, Department of Biology, University of Turku, 20014 Turku, Finland \\ ${ }^{3}$ Department of Ecology, University of Oulu, Pentti Kaiteran katu 1, 90014 Oulu, Finland
}

\begin{abstract}
As a compensatory response to herbivory, plants may branch vigorously when the growth of dormant meristems is triggered by shoot damage. Undamaged plants, on the other hand, often restrain branching, and this limitation on growth can be considered a cost of tolerance to herbivory. Restrained branching is caused by apical dominance and may, alternatively, be associated with fitness benefits in competitive environments that favor fast vertical growth. To test these hypotheses regarding selection for restrained branching, we compared the performance of two subspecies of the biennial grassland herb Gentianella campestris; the tall, apically dominant ssp. campestris and the short, multi-stemmed ssp. islandica, which shows reduced apical dominance. For both subspecies, we manipulated the height of surrounding vegetation (competition) and damage intensity in grasslands of differing productivity (high, medium, low), and examined population growth rates using matrix population models combined with life table response experiments. In the absence of damage, ssp. campestris exhibited a higher population growth rate than ssp. islandica in the tallest vegetation, however with the growth rate still being below one. In the medium and low productivity environments where the vegetation was shorter, the population growth rate of ssp. islandica was considerably higher than that of ssp. campestris as long as no more than about $50 \%$ of the plants were damaged. When plants were damaged, the apically dominant ssp. campestris showed a positive population growth rate $(\lambda>1)$ and often overcompensatory seed production in all productivity levels, while ssp. islandica showed no compensation and therefore the population was predicted to decline $(\lambda<1)$. We conclude that restrained branching in Gentianella cannot be selected for by competition alone, but that episodes of apical damage are required to maintain the trait. Furthermore, because of the costs of restrained branching, apical dominance should be selected against in grasslands where competition and disturbance are low.
\end{abstract}

Key words: apical damage; compensatory growth; demographic model; grassland; herbivory; overcompensation 30 years later; population growth rate.

\section{INTRODUCTION}

Thirty years ago, Paige and Whitham (1987) reported that browsed scarlet gilia (Ipomopsis aggregata) plants produced about 2.4 times more fruits than undamaged plants. Such overcompensation is now known to represent an extreme case in a continuum of plant compensatory responses (or tolerance) to herbivory or other shoot damage (Maschinski and Whitham 1989, Bergelson and Crawley 1992, Strauss and Agrawal 1999, Hawkes and Sullivan 2001, Wise and Abrahamson 2005). Overcompensatory seed production, i.e., when

Manuscript received 27 February 2017; revised 25 October 2017; accepted 1 November 2017. Corresponding Editor: Ken N. Paige.

Editors' Note: Papers in Special Features are published individually and will be linked online in a Collection at: www.wiley. com/go/ecologyjournal

${ }^{4}$ E-mail: tommy.lennartsson@slu.se damaged plants produce more seeds than undamaged ones, has attracted particular attention when it occurs in short-lived species that reproduce only once during their lifetime, partly because it is counterintuitive that such plants do not seem to maximize reproduction in the absence of herbivory (Crawley 1987, Paige and Whitham 1987).

Plant tolerance is one of several adaptations by which plants can persist in environments with high risk of damage by herbivory. The anti-herbivore adaptations are usually classified into three main groups: (1) escape in time or space that reduces the risk of being found or reached by herbivores, and thus the risk of damage, (2) mechanical or chemical defense that reduces the degree of damage when the plant is found and attacked by herbivores; and (3) tolerance mechanisms including compensatory growth after damage that reduce the negative effects of damage of a given intensity once it occurs (Belsky et al. 1993). Such adaptations have in particular 
been studied in grassland habitats in which biomass removal by wild or domesticated herbivores is a fundamental process (Frank et al. 1998) that promote high plant diversity. For example, reduced light competition favors small species, and reduced litter accumulation favors recruitment and short-lived species (Hayes and Holl 2003, Amatangelo et al. 2008). Such conditions, however, come with a high risk of herbivory damage and grassland plants exhibit a variety of anti-herbivore adaptations (Ejvu et al. 2009).

Tolerance and other adaptations to damage are based on combinations of several plant traits (Strauss and Agrawal 1999). The strongest compensatory responses to damage have been found in herbaceous species that have relatively unbranched shoot architecture when intact, but that produce multiple fruiting branches when the apically dominant shoot is damaged (Paige and Whitham 1987, Huhta et al. 2000, Rautio et al. 2005). Two major hypotheses have been proposed for the evolution of restrained branching. According to the compensatory growth hypothesis, restrained branching conserves meristems and resources to be used for regrowth after damage (Crawley 1987, van der Meijden 1990, Vail 1992, Tuomi et al. 1994, Aarssen 1995). The limited branching and lower seed production of undamaged plants can be considered a cost of tolerance in the absence of damage, whereas overcompensation after damage represents the fully realized reproduction potential (Tuomi et al. 1994, Juenger et al. 2000). The evolution of overcompensation can be described in terms of a gambling strategy that is related to the relative probabilities of becoming grazed and avoiding damage (Nilsson et al. 1996). A certain frequency of predictable damage is required for the benefits of restrained branching to outweigh the costs (Crawley 1987, van der Meijden 1990, Vail 1992, Tuomi et al. 1994, Nilsson et al. 1996, Lehtilä 2000).

Another, not mutually exclusive, hypothesis for the selection of restrained branching suggests that it is linked to the fitness benefits of fast vertical growth (instead of branching) in competitive environments, such as in tall, dense vegetation. Aarssen and Irwin (1991) reasoned that vigorous branching following apical damage indicates the costs of apical damage for achieving the benefits of higher competition capacity. In the case of overcompensation, this hypothesis requires that plants do not branch until damage releases suppressed lateral meristems from the apical dominance (for discussion see Aarssen and Irwin 1991, Aarssen 1995, Järemo et al. 1996, Rautio et al. 2005).

The grassland biennial field gentian, Gentianella campestris, exhibits an extreme variation in apical dominance and branching patterns that has motivated the subdivision of the species into two subspecies: the tall, apically dominant ssp. campestris and the short, multistemmed ssp. islandica (Pritchard and Tutin 1972; Appendix S1: Fig. S1). These two subspecies sometimes co-occur, but usually grow in discrete populations. Subspecies campestris is known to overcompensate for experimental and natural damage, with some variation in the degree of tolerance between populations, depending on land-use history (Lennartsson et al. 1997, 1998).

Here, we compare the performance of the two subspecies of G. campestris along environmental gradients of competition (different vegetation heights) and damage (clipping) at three levels of grassland productivity. Using a field experiment combined with population models, we aimed to test under which conditions restrained branching caused by apical dominance in ssp. campestris is advantageous over unrestrained branching in ssp. islandica. A competitive advantage of restrained branching in tall vegetation would support the competition hypothesis, and an increased capacity for compensatory growth after damage would support the compensatory growth hypothesis. We furthermore hypothesized that in the absence of damage and of strong competition, the vigorously branching ssp. islandica would have higher seed production and population growth rate than the apically dominant ssp. campestris, thus that restrained branching would be costly under certain environmental conditions.

\section{Materials And Methods}

\section{Study species}

The field gentian, Gentianella campestris. (L) Börner (Gentianaceae), is an endemic European herb. Two biennial and one annual subspecies have been described, of which we study the two biennials here. The subspecies campestris has an erect growth form, about $15-25 \mathrm{~cm}$ high, whereas ssp. islandica has a multi-stemmed growth form about 5-10 cm high (Appendix S1: Fig. S1). At the flowering stage, intact individuals of ssp. campestris are relatively unbranched, but branching and overcompensatory seed production are common following grazing or mowing (Lennartsson et al. 1997; Appendix S1: Fig. S4). Both subspecies lack vegetative propagation, and have blue or white flowers that are pollinated by bumblebees, although self-fertilization is frequent (Lennartsson 2002). The biennial life-cycle is obligate and all plants form a rosette in the first summer, overwinter as a tap root with a top meristem, and flower in the second summer, dying after that (Fig. 1).

Both subspecies occur in anthropogenic, semi-natural pastures and hay-meadows of unfertilized, semi-dry to mesic types. Such habitats are among the least productive grassland types in the agricultural landscape, but differences in soil moisture, soil type, and climate cause a certain variation in productivity between sites. Subspecies campestris is found over the entire distribution area and ecological range of the species, whereas ssp. islandica is confined to low-productive grasslands in a few montane, northern, and Atlantic parts of the species' distribution area. The intensity of grazing varies considerably between years, mainly depending on stocking density, but is on average lower in low-productive pastures 


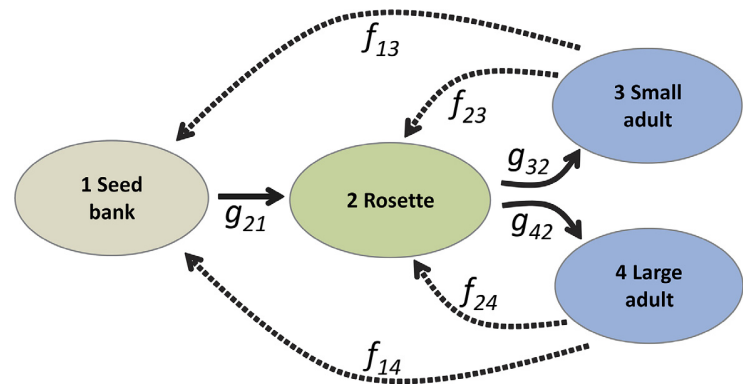

FIG. 1. The life-cycle graph of Gentianella campestris ssp. campestris and ssp. islandica used to construct a $4 \times 4$ matrix population model. The dotted lines indicate fecundity transitions between life stages and the solid lines show growth transitions.

compared to more productive habitats (T. Lennartsson, personal observation).

\section{Experimental setup}

We conducted the experiment in a $75 \times 25 \mathrm{~m}$ area of a semi-natural grassland in central Sweden $\left(59^{\circ} 44^{\prime} 41^{\prime \prime}\right.$ $\mathrm{N}, 18^{\circ} 9^{\prime} 9^{\prime \prime} \mathrm{E}$ ), with dry-to-mesic species-rich herb-grass vegetation (Påhlsson 1994). The grassland sloped slightly (about $4 \%$ incline) from a shallow valley to a low ridge. The soil on the ridge was slightly drier and coarser than in the valley, which created a productivity gradient that was reflected by decreasing vegetation height from the valley to the ridge. Apart from the height gradient the vegetation was homogenous. We used three $10 \times 25 \mathrm{~m}$ sections of the productivity gradient for the experiment, with each section representing a productivity level: high (average vegetation height $15 \mathrm{~cm}$, measured as $50 \%$ visual obstruction method under a $14^{\circ}$ observation angle, Robel et al. 1970), medium (vegetation height about $10 \mathrm{~cm}$ ), or low productivity (vegetation height about $5 \mathrm{~cm}$ ). In August year one, the vegetation in each section was cut and removed together with old litter in order to create optimal conditions for establishment of Gentianella. We then established 18 plots of $2 \times 2 \mathrm{~m}$ in the high productivity section, 12 plots in medium productivity, and six plots in low productivity, in which batches of 4,000 seeds (2,000 seeds per subspecies, randomly mixed) were sown in the $1.6 \times 1.6 \mathrm{~m}$ center (to avoid edge effects). The sowing resulted in 17-31 plants per subspecies per plot (on average 20.6 plants of ssp. campestris and 19.0 of ssp. islandica, of which on average 15.1 and 13.6 plants were assigned to large plants (see section Data sampling). In years two and three, we used a grass shears with an adjustable distance runner to cut the vegetation in the plots to the three heights, representing different levels of competition (see Appendix S1: Fig. S2 for experimental design). In the high productivity section, six plots were cut to a vegetation height of $12 \mathrm{~cm}$, six to $8 \mathrm{~cm}$, and six to $4 \mathrm{~cm}$. In the medium productivity section, six plots were cut to $8 \mathrm{~cm}$ and six to $4 \mathrm{~cm}$ (the vegetation was too short for $12 \mathrm{~cm}$ cutting), and in the low productivity section, six plots were cut to $4 \mathrm{~cm}$ (no $8 \mathrm{~cm}$ or $12 \mathrm{~cm}$ cutting was possible). In both years, the plots were cut three times during the summer (late June, mid-July, and midAugust), in order to keep each plot's experimental vegetation height fairly constant. The cutting of years two and three determined the competition conditions for the rosette and adult stages, respectively. At the June and August cuttings of year three, all adult plants were left untouched, while at the cutting in mid-July, half of the Gentianella plants of each plot were clipped to the same height as plot's vegetation height. We chose plants for clipping by sorting the individuals of each subspecies by size (number of buds) and then alternately assigning plants on the list to clipping or control. Small plants that were shorter than the assigned vegetation height of a given plot were not clipped. By this experimental setup we obtained subsamples of clipped plants subject to different levels of damage and competition, and unclipped plants subject to different levels of competition only.

\section{Data sampling}

At the August-cutting in year two, all rosettes that could be detected with a gentle search were marked using a $2.5 \mathrm{~cm}$ grid, and their diameter and the number of leaves were recorded for each. As rosette survival was nearly $100 \%$, it was possible to assign each rosette to a subspecies retroactively in the third year based on the position of the rosettes and adult plants. At the mid-July cutting in year three, all adult plants of Gentianella were recorded. Small adult plants emanating from small rosettes that had not been detected the previous year, were denoted as "small adults" and all other plants as "large adults" (Fig. 1). The numbers of flower buds, flowers, and fruits were counted in August, and the number of fruits again in late September. Seed production was estimated by multiplying the number of fruits per plant by the number of seeds of a randomly chosen fruit per plant. Seed weight was not considered here because previous studies (Lennartsson 1997, Lennartsson et al. 1997) have shown that seed weight does not differ between the subspecies or between clipped and unclipped plants.

\section{Statistical analyses and tolerance}

In the high productivity section that contained plots of all three experimental vegetation heights, we examined the effects of vegetation height and clipping on the fecundity (seed and bud production) and rosette size of the two subspecies with generalized linear mixed-effects models (function "glmer" in the lme4 package in R 3.2.2, $\mathrm{R}$ Core Team, 2016). In the model of seed production, we included subspecies, vegetation height $(4,8,12 \mathrm{~cm})$, clipping (yes, no), all two-way interactions, and a threeway interaction as fixed explanatory variables. Plot was included as a random effect to account for multiple observations per plot, and the model was fit with the 
negative binomial distribution and log link function Since the three-way interaction (subspecies $\times$ vegetation height $\times$ clipping $)$ was significant $\left(\chi^{2}=48.33, \mathrm{df}=2\right.$, $P<0.0001$ ), we constructed models for unclipped and clipped plants separately to examine seed production in more detail, with vegetation height, subspecies and their interaction as fixed explanatory variables. Moreover, to explore plant responses to clipping within subspecies, we ran models for each subspecies with vegetation height, clipping treatment, and their interaction as fixed explanatory variables. As the clipping treatment did not affect small adult plants, we excluded them from all analyses of the effect of clipping.

To investigate rosette size (number of rosette leaves $x$ rosette diameter in $\mathrm{cm}$ ) in relation to vegetation height, we conducted a glmer with subspecies, vegetation height (three levels), and their interaction as fixed explanatory variables, and with plot as a random effect. Rosette size was modelled using the Gaussian distribution and identity link function.

For the model of bud production, the fixed explanatory variables were subspecies, rosette size from previous year, vegetation height (3 levels), all two-way interactions, and a three-way interaction. Plot was again included as a random effect, and bud production was modelled using the Poisson distribution and log link function.

In all statistical models, the significance of the fixed explanatory variables was explored using the likelihood ratio test fit with maximum likelihood and by testing the model with a given variable against the model without that variable. The goodness of fit was confirmed by visual examination of the residual plots for each model. Moreover, the dispersion factor was examined for the models of seed and bud production (it was close to 1 in both cases, indicating a good fit of the models). Contrasts (function "glht" in the multcomp package) were used for pairwise comparisons for significant explanatory factors, and least square means were calculated using the function "lsmeans" in the lsmeans package.

We quantified tolerance to clipping damage by comparing the mean seed production of the clipped large plants to that of the unclipped large ones separately for each productivity level, subspecies and vegetation height. A ratio less than 1 of clipped to unclipped plants indicates undercompensation, while a ratio greater than 1 indicates overcompensation (Belsky 1986).

\section{Matrix population models}

To examine the consequences of the competition and clipping treatments for the population growth rates of each subspecies, we used a stage-structured matrix population model that consisted of the following four life stages: a short-lived transient seed bank, rosettes, small adults and large adults (see Fig. 1). The matrix model describes the transitions of individual plants from life stages in year $t$ to life stages in year $t+1$, and is appropriate for the studied G. campestris subspecies because they are strictly biennial (i.e., all plants flower in the second year irrespective of plant size; Lennartsson 1997). A transition matrix was constructed separately for each combination of subspecies, productivity level, and clipping treatment, resulting in a total of 12 autumn-autumn matrices per subspecies (Table 1). As the clipping treatments of the adult plants did not influence the rosette stage, we averaged the values of all rosettes per productivity level, subspecies, and vegetation height to calculate the life-cycle transitions related to recruitment and the fate of rosettes $\left(g_{21}, g_{32}, g_{42}\right.$, Fig. 1).

Recruitment for the population matrices could not be properly estimated from the same experiment because the seeds had been sown under optimal recruitment conditions, thus overestimating the rosette/seed ratio. In order to form a litter layer related to each vegetation height, nine additional $2 \times 2 \mathrm{~m}$ plots in the high productivity section were clipped to $12 \mathrm{~cm}, 8 \mathrm{~cm}$, or $4 \mathrm{~cm}$ (three plots/ height) annually in August over three consecutive years (Appendix S1: Fig. S2). In late August of the second year, half of each plot was seeded with 2,000 seeds of ssp. campestris and half with 2,000 seeds of ssp. islandica. The number of rosettes of all sizes was recorded in mid-July in years three and four, which provided, respectively, estimates of recruitment from the previous year's seeds $\left(f_{23}\right.$ and $\left.f_{24}\right)$ and recruitment from the seed bank ( $g_{21}$, Fig. 1). We also examined potential recruitment from the seed bank in year five, but as it was very low (less than $0.01 \%$ for both subspecies), we did not consider a longer-term seed bank in the life-cycle. For each transition matrix, the deterministic population growth rate was calculated as the dominant eigenvalue $(\lambda)$ that describes the rate at which population size is predicted to change in a constant environment (Caswell 2001).

\section{Life table response experiments}

We used a life table response experiment (LTRE; Caswell 2001) to determine the matrix elements (i.e., lifecycle transitions) that contributed to the observed differences in deterministic population growth rates between the clipped and unclipped matrices at each productivity level per subspecies. For details, see Appendix S1.

\section{Stochastic population growth rate relative to damage risk and productivity}

To investigate the long-term stochastic population growth rate $\left(\lambda_{\mathrm{s}}\right)$ with respect to productivity and simulated grazing intensity, we conducted stochastic simulations for each productivity level (high, medium, low). For each subspecies, we used sets of the transition matrices constructed from a given productivity level (Table 1) and simulated population size for $10,000 \mathrm{yr}$ starting from 50 individuals in each life stage. The clipped matrices ("cl" in Table 1) in a given simulation were drawn at a probability of $0,0.2,0.4,0.6,0.8$, or 1.0 , and the unclipped matrices 
TABle 1. Demographic transitions (see life-cycle in Fig. 1) and annual population growth rate ( $\lambda$ ) for the unclipped (uc) and clipped (cl) populations of Gentianella ssp. campestris (cam) and islandica (isl) growing in environments that differed in productivity (high, $\mathrm{H}$; medium, $\mathrm{M}$; low, L) and competition (12, 8, $4 \mathrm{~cm}$ vegetation height). Growth transitions are $g_{21}$ (seeds in the seed bank developing into rosettes), $g_{32}$, and $g_{42}$ (rosettes developing into adults). Fecundity transitions are $f_{14}, f_{13}$ (seed production to the seed bank), $f_{23}, f_{24}$ (production of new rosettes).

\begin{tabular}{|c|c|c|c|c|c|c|c|c|}
\hline \multirow[b]{2}{*}{ Matrix no. and code } & \multirow[b]{2}{*}{$\lambda$} & \multicolumn{7}{|c|}{ Transitions } \\
\hline & & $g_{21}$ & $g_{32}$ & $g_{42}$ & $f_{13}$ & $f_{23}$ & $f_{14}$ & $f_{24}$ \\
\hline 1 [H 12 cam uc] & 0.567 & 0.00017 & 0.420 & 0.558 & 150.2 & 0.165 & 299.8 & 0.330 \\
\hline 2 [H 12 isl uc] & 0.440 & 0.00015 & 0.397 & 0.575 & 114.8 & 0.149 & 126.2 & 0.164 \\
\hline $3[\mathrm{H} 12 \mathrm{cam} \mathrm{cl}]$ & 0.530 & 0.00017 & 0.420 & 0.558 & 150.2 & 0.165 & 243.3 & 0.268 \\
\hline 4. [H 12 isl cl] & 0.446 & 0.00015 & 0.397 & 0.575 & 114.8 & 0.149 & 131.9 & 0.171 \\
\hline 5 [H 8 cam uc] & 1.031 & 0.00031 & 0.191 & 0.809 & 131.2 & 0.276 & 517.7 & 1.087 \\
\hline 6 [H 8 isl uc] & 1.338 & 0.00032 & 0.176 & 0.820 & 195.2 & 0.390 & 932.7 & 1.865 \\
\hline 7 [H 8 cam cl] & 1.220 & 0.00031 & 0.191 & 0.809 & 131.2 & 0.276 & 752.5 & 1.580 \\
\hline $8[\mathrm{H} 8$ isl $\mathrm{cl}]$ & 0.832 & 0.00032 & 0.176 & 0.820 & 195.2 & 0.390 & 312.0 & 0.624 \\
\hline 9 [H 4 cam uc] & 1.188 & 0.00036 & 0.108 & 0.890 & 227.2 & 0.523 & 581.8 & 1.338 \\
\hline 10 [H 4 isl uc] & 1.800 & 0.00034 & 0.120 & 0.880 & 363.8 & 0.873 & 1,373 & 3.296 \\
\hline $11[\mathrm{H} 4 \mathrm{cam} \mathrm{cl}]$ & 1.202 & 0.00036 & 0.108 & 0.890 & 49.10 & 0.112 & 617.4 & 1.420 \\
\hline $12[\mathrm{H} 4$ isl $\mathrm{cl}]$ & 0.543 & 0.00034 & 0.120 & 0.881 & 40.90 & 0.098 & 105.2 & 0.252 \\
\hline 13 [M 8 cam uc] & 0.946 & 0.00031 & 0.230 & 0.770 & 105.8 & 0.223 & 448.0 & 0.941 \\
\hline 14 [M 8 isl uc] & 1.201 & 0.00032 & 0.241 & 0.759 & 148.3 & 0.311 & 755.1 & 1.586 \\
\hline 15 [M 8 cam cl] & 1.113 & 0.00031 & 0.230 & 0.770 & 105.8 & 0.223 & 542.1 & 1.382 \\
\hline 16 [M 8 isl cl] & 0.876 & 0.00032 & 0.241 & 0.759 & 148.3 & 0.311 & 363.4 & 0.762 \\
\hline 17 [M 4 cam uc] & 1.084 & 0.00036 & 0.160 & 0.844 & 184.6 & 0.426 & 497.4 & 1.143 \\
\hline 18 [M 4 isl uc] & 1.567 & 0.00034 & 0.180 & 0.820 & 317.2 & 0.729 & 1,120 & 2.576 \\
\hline 19 [M 4 cam cl] & 1.082 & 0.00031 & 0.160 & 0.844 & 44.90 & 0.103 & 521.3 & 1.198 \\
\hline 20 [M 4 isl cl $]$ & 0.750 & 0.00032 & 0.180 & 0.820 & 34.12 & 0.078 & 242.5 & 0.557 \\
\hline 21 [L 4 cam uc] & 0.992 & 0.00036 & 0.211 & 0.797 & 153.7 & 0.354 & 424.3 & 0.975 \\
\hline 22 [L 4 isl uc] & 1.445 & 0.00034 & 0.208 & 0.790 & 267.2 & 0.614 & 971.0 & 2.233 \\
\hline 23 [L 4 cam cl] & 1.016 & 0.00036 & 0.211 & 0.797 & 38.88 & 0.094 & 459.5 & 1.102 \\
\hline 24 [L 4 isl cl] & 0.712 & 0.00034 & 0.208 & 0.790 & 30.02 & 0.072 & 214.9 & 0.516 \\
\hline
\end{tabular}

at the inverse probabilities. We assumed an equal likelihood for being grazed to $4 \mathrm{~cm}$ or $8 \mathrm{~cm}$. For example, for the simulation of $40 \%$ damage risk of ssp. campestris in the high productivity environment, we drew the unclipped matrix no. 1 in Table 1 at a probability of 0.6 and the two clipped matrices no. 7 and 11 at a probability of 0.2 each. For each productivity level, we then calculated the average stochastic population growth rate with $95 \%$ confidence intervals across time steps based on population sizes; the first 1,000 yr were removed to omit transient dynamics. To facilitate comparison with the deterministic growth rates, we present all stochastic growth rates with the same scale, i.e., $\lambda_{\mathrm{s}}>1$ indicates an increasing population and $\lambda_{\mathrm{s}}<1$ indicates a declining population in a variable environment.

\section{REsUlTS}

\section{Effects of vegetation height on plant performance in the absence of damage}

The effects of vegetation height on seed production differed between the two subspecies: seed production of the unclipped ssp. islandica plants was considerably higher than that of ssp. campestris plants in $4 \mathrm{~cm}$ and $8 \mathrm{~cm}$ vegetation, while the opposite was true in $12-\mathrm{cm}-$ tall vegetation $\left(\chi^{2}=37.66, \mathrm{df}=2, P<0.0001\right.$ for the subspecies $\times$ vegetation height interaction; Fig. 2a).

For both subspecies, rosette size decreased with increasing vegetation height $\left(\chi^{2}=56.91, \quad \mathrm{df}=1\right.$, $P<0.0001$ for vegetation height and $\chi^{2}=2.23, \mathrm{df}=2$, $P=0.329$ for the subspecies $\times$ vegetation height interaction; Fig. 3a). The rosette size in turn correlated strongly with the bud production of the following year's adult plants, but this relationship differed between the subspecies across vegetation heights $\left(\chi^{2}=7.69, \mathrm{df}=1\right.$, $P=0.006$ for the subspecies $\times$ rosette size interaction and $\chi^{2}=116.54, \mathrm{df}=2, \quad P<0.0001$ for the subspecies $\times$ vegetation height interaction). In the two lowest vegetation heights, ssp. islandica produced more buds in relation to rosette size than ssp. campestris, but in the tallest vegetation, there was no such a difference (Fig. 3b). The negative LTRE contributions of rosette recruitment indicate recruitment limitation in tall vegetation (Appendix S1: Fig. S3).

For both subspecies, $\lambda>1$ predicted unclipped populations to increase in $4 \mathrm{~cm}$ and $8 \mathrm{~cm}$ vegetation, while $\lambda<1$ predicted unclipped populations to decline in the tallest $(12 \mathrm{~cm})$ vegetation (Table 1 , compare matrices 5 , $6,9,10$ with matrices 1,2$)$. In $4 \mathrm{~cm}$ and $8 \mathrm{~cm}$ vegetation, ssp. islandica had a higher population growth rate than ssp. campestris in the absence of damage (Table 1, 


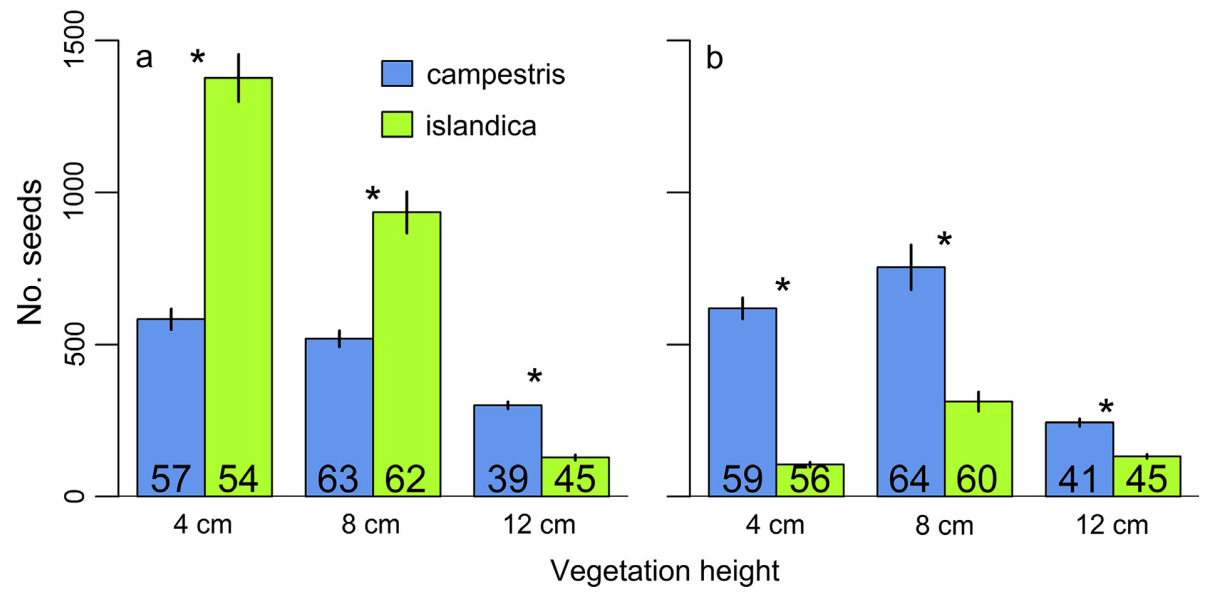

FIG. 2. Seed production for (a) unclipped and (b) clipped large plants of Gentianella campestris ssp. campestris and ssp. islandica growing in three vegetation heights (least square mean $\pm \mathrm{SE}$ ). Sample sizes are shown on the bars and significant differences $(P<0.05$ based on contrasts) between the subspecies are indicated with a star.

matrices 6 vs. 5 and 10 vs. 9). This difference between subspecies was mainly due to ssp. islandica's greater seed and rosette production, i.e., transitions $f_{23}$ and $f_{24}$ (Fig. 2a; Appendix S1: Fig. S3). Moreover, the population growth rate of both subspecies increased with increasing productivity level (Table 1, compare matrices 9, 17, 21 for ssp. campestris and matrices 10, 18, 22 for ssp. islandica).

\section{Effects of clipping on plant performance}

When the plants were clipped, the seed production of ssp. campestris was significantly higher than that of ssp. islandica in all vegetation heights, with the difference between the subspecies being most pronounced in $8-\mathrm{cm}$ tall vegetation $\left(\chi^{2}=18.97, \mathrm{df}=2, P<0.0001\right.$ for the
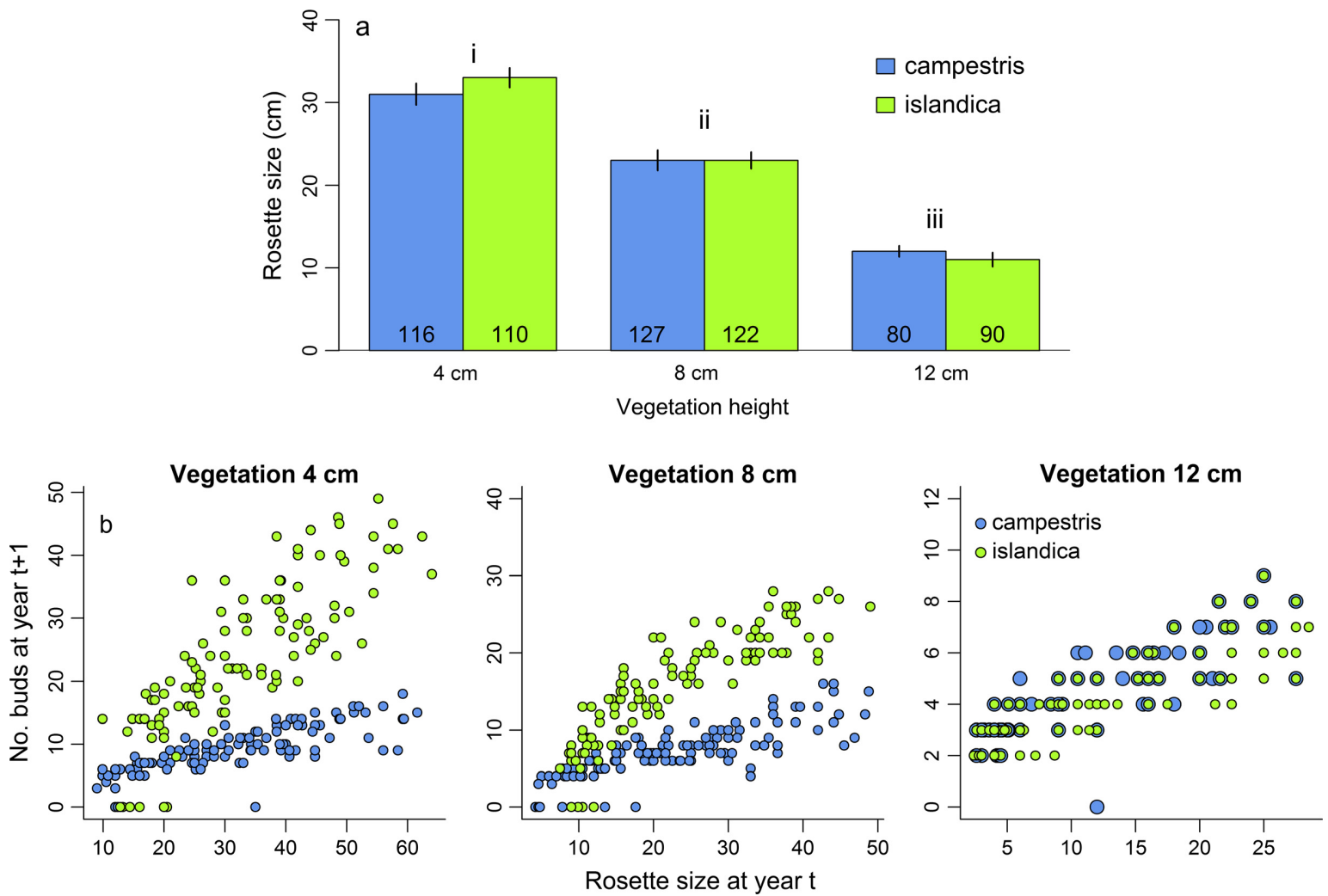

FIG. 3. (a) Rosette size (number of leaves $\times$ diameter in $\mathrm{cm}$; least square mean $\pm \mathrm{SE}$ ) and (b) Number of flower buds relative to rosette size in different vegetation heights for Gentianella campestris ssp. campestris and ssp. islandica. In (a) $i$-iii indicate significant differences $(P<0.05$ based on contrasts) between vegetation heights. 
subspecies $\times$ vegetation height interaction; Fig. 2b). Subspecies campestris overcompensated for clipping in the $4 \mathrm{~cm}$ and $8 \mathrm{~cm}$ vegetation (ratio of seed production of the clipped and unclipped plants $=1.06$ for $4 \mathrm{~cm} ; 1.45$ for $8 \mathrm{~cm}$ ), but undercompensated for clipping in 12-cm-tall vegetation (ratio $=0.81, P<0.05$ in all cases based on contrasts; Fig. 2). Subspecies islandica undercompensated for clipping in the $4 \mathrm{~cm}$ and $8 \mathrm{~cm}$ vegetation (ratio $=0.08$ and 0.33 , respectively, $P<0.05$ in both cases, contrasts), while clipping at $12 \mathrm{~cm}$ removed rather few buds in this low-growing subspecies and caused no difference from the unclipped plants ( $P=0.255$, contrasts; Fig. 2$)$.

The compensatory growth of ssp. campestris after clipping led to a positive population growth rate $(\lambda>1)$ in the $4 \mathrm{~cm}$ and $8 \mathrm{~cm}$ vegetation, while clipping of ssp. islandica caused a negative growth rate $(\lambda<1$; Table 1 , compare ssp. campestris matrices 7 and 11 with ssp. islandica matrices 8 and 12). Clipping to $12 \mathrm{~cm}$ resulted in declining populations for both subspecies (Table 1, matrices 3 and 4). The LTRE analysis indicated that these population declines were due to dramatic reductions in rosette production, (Appendix S1: Fig. S3). Overall, the LTRE analysis confirmed that the effect of clipping on population growth rate varied between subspecies and depended on the productivity level and clipping treatment in question, as indicated by large contributions for the subspecies $\times$ clipping interaction (Appendix S1: Fig. S3).

\section{Stochastic population growth rate in gradients of damage and productivity}

In stochastic simulations ssp. islandica formed viable populations $\left(\lambda_{\mathrm{s}}>1\right)$ when the damage risk was low $(<50 \%$ of plants being damaged) in the medium- and low-productivity environments (Fig. 4). In the most productive environment, $\lambda_{\mathrm{s}}$ of ssp. islandica increased with increasing frequency of grazed patches, but never reached growth rate $>1$ (Fig. 4). In contrast, ssp. campestris showed a positive population growth rate under conditions of high damage risk in the high-productivity $(>80 \%$ damage risk) and medium-productivity ( $>40 \%$ damage risk) environments, and about stable population dynamics $\left(\lambda_{\mathrm{s}}=1\right)$ in the low productivity environment regardless of damage risk (Fig. 4).

\section{Discussion}

\section{Apical dominance and restrained growth for tolerance or competition?}

Our comparison of the two Gentianella campestris subspecies showed that the apically dominant ssp. campestris was highly tolerant to shoot damage and less sensitive to tall vegetation. On average, damaged plants of this subspecies overcompensated for the damage in terms of seed production and had positive stochastic population growth rate in the productive grassland when $>80 \%$ of the
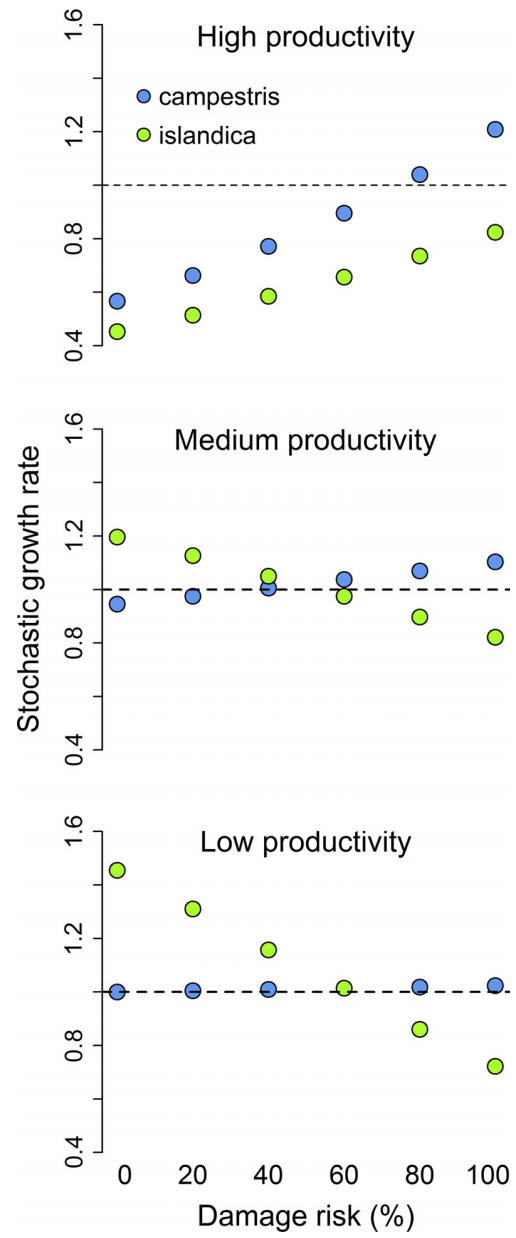

FIG. 4. Stochastic population growth rates (mean $\pm 95 \%$ CI) for Gentianella campestris ssp. campestris and ssp. islandica relative to damage risk at different productivity levels calculated over 10,000 time steps. The population is predicted to decline under the dotted line and increase above it. Note that CIs are small and therefore not visible at the current scale.

area was clipped to short vegetation, corresponding to $80 \%$ of the adult plants being damaged. The branchy ssp. islandica undercompensated for the damage and was predicted to decline in the high-productive grassland in spite of reduced vegetation height. When the plants were undamaged, ssp. campestris produced more seeds and exhibited a higher population growth rate than ssp. islandica only in the tallest $(12 \mathrm{~cm})$ vegetation, indicating an advantage of an erect growth form in shady environments. In the absence of damage and in lower vegetation, ssp. islandica had higher seed production and population growth rate, suggesting a cost of restrained branching under low damage risk and low light competition.

The differential compensatory responses of the two subspecies support the hypothesis that apical dominance and restrained branching are selected for as a tolerance mechanism in environments with a high risk of damage (Crawley 1987, Tuomi et al. 1994), such as grazed or 
mown grasslands (Lennartsson et al. 1997). On the other hand, the differential responses to tall vegetation support the alternative hypothesis, i.e., that apical dominance and fast vertical growth are selected for in productive habitats, in which tall plants may more efficiently compete for light and/or pollinators (Aarssen and Irwin 1991, Aarssen 1995). However, stochastic simulations indicate that this competitive advantage is not sufficient to maintain viable populations (having $\lambda_{\mathrm{s}}>1$ ) of ssp. campestris in grasslands that are constantly high-competitive. The reason is poor rosette growth, in turn reducing the size of adult plants, and poor recruitment, probably as a result of litter accumulation (e.g., Lennartsson and Oostermeijer 2001). In contrast, simulations indicate that tolerance to damage enables viable populations in grasslands that are constantly grazed and thus low-competitive. These results suggest that selection for tolerance is more important in this species than selection for competitive capacity, and that apical dominance and restrained growth may thus be predominantly a mechanism for tolerance.

The competition capacity of ssp. campestris may nevertheless contribute to the selection for apical dominance if grazing is patchy and spatially variable between years. Under such non-constant conditions, a proportion of adult plants will experience high-competitive vegetation, and vertical growth will then enhance the total seed production and growth rate of the population. Both theoretical and empirical studies have shown that spatial and temporal environmental variations enhance population persistence even in poor quality (sink) habitats (Jansen and Yoshimura 1998, Matthews and Gonzalez 2007).

Selection for apical dominance may also be mediated by pollination, as plant height influences pollen transfer (Donnelly et al. 1998, Ehrlén et al. 2002) that, in turn, influences seed set and paternal fitness (Paige et al. 2001). Pollen limitation is, however, not likely in the highly self-pollinating G. campestris, which experiences little (if any) reduction of seed set when pollinators are excluded (Lennartsson 2002).

\section{Tolerance and grassland habitats}

The field gentian studied here has several traits in common with other grassland annuals and biennials, and our results therefore have implications for the interpretation of grassland plant traits in general. Specifically, similar to the field gentian, many short-lived grassland plants can rarely grow tall enough to overtop more competitive perennial neighbors, and can therefore be assumed to exhibit poor growth and performance of all life stages in tall vegetation (Grime 1979, Mooney et al. 1986, Kelly 1989, Amatangelo et al. 2008). These plant species thus require low-competitive habitats for persistence. In grasslands maintained by grazing, such habitats come with a high risk of damage of adult plants, which usually affects seed production negatively (Hayes and Holl 2003). Both seed limitation and recruitment limitation have been found in grasslands (Jacobsson and Eriksson 2000), and population viability of short-lived grassland plants can be expected to be strongly influenced by a tradeoff between the positive effects of intense grazing on early life stages (due to improved recruitment) and negative effects on adults and fecundity (due to increased risk of being damaged). This tradeoff should lead to strong selection for tolerance and other anti-herbivore adaptations to the disturbance in many short-lived species that inhabit grasslands maintained by grazing or mowing (Rosentahl and Kotanen 1994). In such species, apical dominance may be more important as a tolerance mechanism than as a competitive advantage. Although compensatory seed production following apical damage has been observed in several grassland annuals and biennials (Huhta et al. 2003), the relative advantage of tolerance and competition capacity in most cases remains to be studied.

In grasslands where the turf height is limited by nutrient deficit or similar stress factors rather than by intense disturbance, selection for unrestrained branching should occur because of the cost of apical dominance under low competition and low risk of damage (Irwing and Aarssen 1996a, Crawley 1987). For example, Gentianella campestris ssp. islandica and a number of other grassland species including the Gentianella amarella and G. germanica groups, Rhinanthus, and Euphrasia, are known to have branchy types at high altitudes (Pritchard and Tutin 1972, Westbury 2004). High-altitude ecotypes are usually interpreted as adaptations to harsh environments, but we propose that they in some cases represent selection for reduced apical dominance in short-vegetation grasslands that are maintained without intense summer disturbance.

In the case of the field gentian, ssp. islandica occurs in Scandinavia only in northern or heathland pastures that are very low-productive due to poor soils and a short growing season (Pritchard and Tutin 1972). Low-productive grasslands support few grazers, and, as a consequence, gentians in such habitats experience rather little herbivory (usually less than $20 \%$ of the plants are damaged; T. Lennartsson, personal observation). In more productive pastures, where most populations of ssp. campestris occur, the frequency of damaged gentian plants is on average considerably higher, but often varies greatly between years from 20 to $100 \%$ depending on the farmer's choice of stocking density, type of grazer, timing of grazing, and duration of the grazing period (Lennartsson 1997). In hay meadows, mowing damages nearly $100 \%$ of the plants. The difference in damage frequency between low-productive and more productive sites is probably consistent enough to cause different selection pressures on the branching pattern. Both subspecies studied in the present study originated from populations (one per subspecies) with a long history of grazing, although differing in intensity due to differing site productivity as described above. Previously, Lennartsson et al. (1997) found differences in tolerance also between populations of ssp. campestris also in more productive grasslands; populations from pastures and hay meadows overcompensated for 
damage, whereas populations from unmanaged road verges and powerline corridors did not. Grazing-induced differentiation between ssp. campestris populations is, however, probably limited by the large between-year variation in damage frequency in productive pastures.

\section{Compensatory growth in experiments and nature}

In this study we used clipping and stochastic matrix modelling to imitate and simulate grassland disturbance, and the results need to be evaluated considering the similarities and differences between the experiment and natural conditions (Paige 1994). Clipping is the most commonly used experimental treatment to imitate herbivory, and has proven to give largely the same responses as shoot damage found in nature, provided that the degree and timing of damage are accurate (Paige and Whitham 1987, Strauss and Agrawal 1999). In pastures, grazing normally removes $40-80 \%$ of the shoot length of ssp. campestris and frequently trigs the same pattern of compensatory growth as our experimental clipping (T. Lennartsson, personal observation; Appendix S1: Fig. S4). We therefore consider that our experimental clipping represents a realistic type of damage. Moreover, grazing normally produces patchy vegetation in which the frequency of grazed patches increases with grazing intensity (Adler et al. 2001), i.e. similar to our stochastic simulations.

The degree of damage plays an important role in plant compensatory responses and, consequently, estimates of tolerance (McNaughton 1979, Belsky 1986, Huhta et al. 2003). In our study, the plants compensated best at intermediate damage levels (about half of the shoot removed). This is consistent with the theory that the damage on the one hand needs to leave enough resources and meristems for regrowth, but on the other hand needs to remove enough of the apical suppression of basal meristems (Sachs 1999, Huhta et al. 2000, Klimešová et al. 2014).

Also the timing of damage in relation to a species' phenology influences compensation capacity. Paige (1994) showed that plants of Ipomopsis aggregata clipped early in the season compensated better than those clipped later, and related this result to the phenology of stem elongation and bud development (Maschinski and Whitham 1989). Gentianella campestris partly shows the same pattern, i.e. damage closer to the date of flowering reduces the compensatory response. In this species, however, also very early clipping reduces compensation, probably because the below-ground resources become exhausted during the initial growth of the adult plant in the spring. Our clipping in mid-July corresponds to the time window of overcompensation identified in an earlier study (Lennartsson et al. 1998).

\section{Tolerance and resources}

Compensatory growth after damage is often fueled by carbon stored in roots (Strauss and Agrawal 1999). Although we have no data on taproot weight of adult plants of the two Gentianella types, ssp. islandica can be assumed to allocate most of the taproot resources to adult growth, whereas ssp. campestris should save some resources for regrowth, either by restraining the allocation to shoots in the spring or by re-storing resources in the early summer. Both subspecies are probably maximizing resource storage during the rosette stage, and preliminary studies have shown that the tap root dry weight does not differ between subspecies when matched by rosette size (T. Lennartsson, unpublished data).

Based on the light competition hypothesis, Irwing and Aarssen (1996b) proposed that plants compensate best in environments with intermediate nutrient levels, and a meta-analysis by Hawkes and Sullivan (2001) showed that dicots compensate better in low than in high resource conditions. In our study, however, the average compensation of ssp. campestris (i.e. the ratio of seed production of clipped/unclipped plants) was positively correlated with productivity. This was due to a larger proportion of small, poorly compensating plants in low productivity (transition $g_{32}$ in Table 1), whereas the compensation of large plants $\left(f_{14}\right)$ was approximately even across productivity levels. Our study area probably represents only the intermediate to low end of a nutrient gradient, but it is also possible that our result reflects selection for tolerance rather than for competitive advantages. Under such selection, a certain proportion of the resources should be saved for regrowth irrespective of nutrient availability.

\section{Benefits and costs of adaptations to herbivory}

Even at maximum overcompensation, the population growth rate of ssp. campestris in this study was lower than that of undamaged ssp. islandica (only in $12 \mathrm{~cm}$ vegetation, damaged ssp. campestris plants had higher seed production than undamaged ssp. islandica plants, but in such tall vegetation, the population growth rate of both subspecies was far below one). This observation is consistent with theoretical models of the evolution of overcompensation, for example, resource and meristem allocation models (Vail 1992, Tuomi et al. 1994), which predict that the absolute fitness of a damaged and overcompensating grazing-adapted type will remain below that of an ungrazed non-compensating "ancestor" type that has evolved in the absence of herbivores (Mathews 1994, Tuomi et al. 1994). Subspecies islandica in this study represents such a non-compensating control and, to our knowledge, provides the first empirical evidence of the predictions of the resource and meristem allocation models. In the models, the absolute fitness of overcompensators is reduced due to allocation costs of regrowth potential and because the damage that induces regrowth causes a certain loss of meristems and resources (Tuomi et al. 1994, see also Simons et al. 2007). In addition, the compensatory regrowth often leads to delayed flowering, which can reduce seed set in time-limited seasonal environments (Piippo et al. 2009).

Overcompensation is sometimes considered a case of plant-herbivore mutualism (Paige and Whitham 1987, 
Vail 1992, Agrawal 2000, but see Belsky et al. 1993), and our results can be discussed in a mutualism context. Overcompensation can be regarded an example of evolved dependence, i.e., that the plant through adaptation to a herbivore partner has compromised its ability to perform well in the absence of the partner (de Mazancourt et al. 2005). If ssp. islandica is viewed as an evolutionary ancestor of ssp. campestris, our study indicates that the overcompensation of ssp. campestris does not represent an ultimate benefit of herbivory in the sense of increased plant performance over evolutionary time (Järemo et al. 1999, de Mazancourt et al. 2005). The evolutionary benefits of grazing, however, also depend on indirect effects of grazing via changes in community structure and ecosystem processes (Crawley 1987, de Mazancourt and Loreau 2000, de Mazancourt et al. 2001), because the grazers function as niche constructors (Eriksson 2013). Just as the savanna grasses discussed by McNaughton (1979, 1986), Gentianella campestris ssp. campestris can be considered to be evolutionary dependent on, and favored by, herbivory since its habitat would not exist without grazing.

\section{Concluding remarks}

Similar to previous studies (Ehrlén 2003, 2015), the present study emphasizes that the entire life-cycle of the study species must be taken into account when assessing the benefits of tolerance mechanisms and other plant adaptations in grassland habitats. When doing that, our results indicate that the grazing-tolerant ssp. campestris has evolved as an adaptation to grazing either in the presence or absence of episodes of competition, and that selection for apical dominance is a fundamental component of that evolution. We acknowledge that the evolution of apical dominance in most plant species represents an evolutionary response to competitive environments (Aarssen and Irwin 1991), but suggest that in grassland habitats that are shaped by intense grazing, apical dominance may be an important and overlooked mechanism for tolerance.

\section{ACKNOWLEDGMENTS}

We thank two anonymous reviewers and the editor for valuable comments and suggestions. This study was financially supported by the Swedish Research Council FORMAS (award 34.0297 to TL) and the Academy of Finland (grant 285746 to SR).

\section{Literature Cited}

Aarssen, L. W. 1995. Hypothesis for the evolution of apical dominance in plants: implications for the interpretation of overcompensation. Oikos 74:149-156.

Aarssen, L. W., and D. L. Irwin. 1991. What selection: herbivory or competition? Oikos 60:261-262.

Adler, P. B., D. A. Raff, and W. K. Lauenroth. 2001. The effect of grazing on the spatial heterogeneity of vegetation. Oecologia 128:465-479.

Agrawal, A. A. 2000. Overcompensation of plants in response to herbivory and the by-product benefits of mutualism. Trends in Plant Science 5:309-313.
Amatangelo, K., K. L. Dukes, S. Jeffrey, and C. B. Field. 2008. Responses of a California annual grassland to litter manipulation. Journal of Vegetation Science 19:605-612.

Belsky, A. J. 1986. Does herbivory benefit plants? A review of the evidence. American Naturalist 127:870-892.

Belsky, A. J., W. P. Carson, C. L. Jensen, and G. A. Fox. 1993. Overcompensation by plants: herbivore optimization or red herring? Evolutionary Ecology 7:109-121.

Bergelson, J., and M. J. Crawley. 1992. The effects of grazers on the performance of individuals and populations of scarlet gilia, Ipomopsis aggregata. Oecologia 90:435-444.

Caswell, H. 2001. Matrix population models: construction, analysis, and interpretation. Sinauer Associates, Sunderland, Massachusetts, USA.

Crawley, M. J. 1987. Benevolent herbivores? Trends in Ecology and Evolution 2:167-168.

de Mazancourt, C., and M. Loreau. 2000. Grazing optimization, nutrient cycling, and spatial heterogeneity of plantherbivore interactions: should a palatable plant evolve? Evolution 54:81-92.

de Mazancourt, C., M. Loreau, and U. Dieckmann. 2001. Can the evolution of plant defense lead to plant-herbivore mutualism? American Naturalist 158:109-123.

de Mazancourt, C., M. Loreau, and U. Dieckmann. 2005. Understanding mutualism when there is adaptation to the partner. Journal of Ecology 93:305-314.

Donnelly, S. E., C. J. Lortie, and L. W. Aarssen. 1998. Pollination in Verbascum thapsus (Scrophulariaceae): The advantage of being tall. American Journal of Botany 85:1618-1625.

Ehrlén, J. 2003. Fitness components versus total demographic effects: evaluating herbivore impacts on a perennial herb. American Naturalist 162:796-810.

Ehrlén, J. 2015. Selection on flowering time in a life-cycle context. Oikos 124:92-101.

Ehrlén, J., S. Käck, and J. Ågren. 2002. Pollen limitation, seed predation, and scape length in Primula farinosa. Oikos 97:45-51.

Ejvu, M., G. Austrheim, R. Halvorsen, and A. Mysterud. 2009. Grazing responses in herbs in relation to herbivore selectivity and plant traits in an alpine ecosystem. Oecologia 161:77-85.

Eriksson, O. 2013. Species pools in cultural landscapes - niche construction, ecological opportunity and niche shifts. Ecography 36:403-413.

Frank, D. A., S. J. McNaughton, and B. F. Tracy. 1998. The ecology of the Earth's grazing systems. BioScience 48:513-521.

Grime, J. P. 1979. Plant strategies and vegetation processes. John Wiley \& Sons, Chichester, UK.

Hawkes, C. V., and J. J. Sullivan. 2001. The impact of herbivory on plants in different resource conditions: a meta-analysis. Ecology 82:2045-2058.

Hayes, G. F., and K. D. Holl. 2003. Cattle grazing impacts on annual forbs and vegetation composition of mesic grasslands in California. Conservation Biology 17:1694-1702.

Huhta, A.-P., T. Lennartsson, J. Tuomi, P. Rautio, and K. Laine. 2000. Tolerance of Gentianella campestris in relation to damage intensity: an interplay between apical dominance and herbivory. Evolutionary Ecology 14:373-392.

Huhta, A. P., K. Hellström, P. Rautio, and J. Tuomi. 2003. Grazing tolerance of Gentianella amarella and other monocarpic herbs: why is tolerance highest at low damage levels? Plant Ecology 166:49-61.

Irwing, D. L., and L. W. Aarssen. 1996a. Testing cost of apical dominance in vegetation: a field study of three species. Annales Botanici Fennici 33:123-128.

Irwing, D. L., and L. W. Aarssen. 1996b. Effects of nutrient level on cost and benefit of apical dominance in Epilobium ciliatum. American Midland Naturalist 136:14-28. 
Jacobsson, A., and O. Eriksson. 2000. A comparative study of seed number, seed size, seedling size and recruitment in grassland plants. Oikos 88:494-502.

Jansen, V. A. A., and J. Yoshimura. 1998. Populations can persist in an environment consisting of sink habitats only. Proceedings of the National Academy of Sciences of the United States of America 95:3696-3698.

Järemo, J., P. Nilsson, and J. Tuomi. 1996. Plant compensatory growth: herbivory or competition? Oikos 77:238-247.

Järemo, J., J. Tuomi, P. Nilsson, and T. Lennartsson. 1999. Plant adaptations to herbivory: mutualistic versus antagonistic coevolution. Oikos 84:313-320.

Juenger, T., T. Lennartsson, and J. Tuomi. 2000. The evolution of tolerance to damage in Gentianella campestris: natural selection and the quantitative genetics of tolerance. Evolutionary Ecology 14:393-419.

Kelly, D. 1989. Demography of short-lived plants in chalk grassland. II. Control of mortality and fecundity. Journal of Ecology 77:770-784.

Klimešová, J., L. Malíková, J. Rosentahl, and P. Šmilauer. 2014. Potential bud bank responses to apical meristem damage and environmental variables: matching or complementing axillary meristems? PLoS ONE 9:e88093. https://doi.org/10.1371/jour nal.pone. 0088093

Lehtilä, K. 2000. Modelling compensatory regrowth with bud dormancy and gradual activation of buds. Evolutionary Ecology 14:315-330.

Lennartsson, T. 1997. Demography, reproductive biology and adaptive traits in Gentianella campestris and G. amarella.Evaluating grassland management for conservation by using indicator plant species. Acta Universitatis Agriculturae Suecicae - Agraria 46. Doctoral Thesis.

Lennartsson, T. 2002. Extinction thresholds and disrupted plant-pollinator interactions in fragmented plant populations. Ecology 83:3060-3072.

Lennartsson, T., and J. G. P. Oostermeijer. 2001. Demographic variation and population viability in Gentianella campestris: effects of grassland management and environmental stochasticity. Journal of Ecology 89:451-463.

Lennartsson, T., J. Tuomi, and P. Nilsson. 1997. Evidence for an evolutionary history of overcompensation in the grassland biennial Gentianella campestris (Gentianaceae). American Naturalist 149:1147-1155.

Lennartsson, T., P. Nilsson, and J. Tuomi. 1998. Induction of overcompensation in the field gentian, Gentianella campestris. Ecology 79:1061-1072.

Maschinski, J., and T. G. Whitham. 1989. The continuum of plant responses to herbivory: the influence of plant association, nutrient availability and timing. American Naturalist 134:1-19.

Mathews, J. N. A. 1994. The benefits of overcompensation and herbivory: the difference between coping with herbivores and liking them. American Naturalist 144:528-533.

Matthews, D. P., and A. Gonzalez. 2007. The inflationary effects of environmental fluctuations ensure the persistence of sink metapopulations. Ecology 88:2848-2856.

McNaughton, S. J. 1979. Grazing as an optimization process: grass-ungulate relationships in the Serengeti. American Naturalist 113:691-703

McNaughton, S. J. 1986. On plants and herbivores. American Naturalist 128:765-777.
Mooney, H. A., R. J. Hobbs, J. Gorham, and K. Williams. 1986. Biomass accumulation and resource utilization in cooccurring grassland annuals. Oecologia 70:555-558.

Nilsson, P., J. Tuomi, and M. Aström. 1996. Bud dormancy as a bet-hedging strategy. American Naturalist 147:269-281.

Påhlsson, L. 1994. Vegetationstyper i Norden. The Nordic Council of Ministers, Copenhagen, Denmark.

Paige, K. 1994. Herbivory and Ipomopsis aggregata: Differences in response, differences in experimental protocol: A reply to Bergelson and Crawley. American Naturalist 143:739-749.

Paige, K. N., and T. G. Whitham. 1987. Overcompensation in response to mammalian herbivory: the advantage of being eaten. American Naturalist 129:407-416.

Paige, K. N., B. Williams, and T. Hickox. 2001. Overcompensation through the paternal component of fitness in Ipomopsis arizonica. Oecologia 128:72-76.

Piippo, S., K. Hellström, A.-P. Huhta, P. Rautio, and J. Tuomi. 2009. Delayed flowering as a potential benefit decreasing cost of compensatory regrowth. Botany-Botanique 87:837-844.

Pritchard, N. M., and T. G. Tutin. 1972. Gentianella Moench. Pages 63-67 in T. G. Tutin, V. H. Heywood, N. A. Burges, D. M. Moore, D. H. Valentine, S. M. Walters, and D. A. Webb, editors. Flora Europaea. Volume 3. Cambridge University Press, Cambridge, UK.

R Core Team. 2016. R: A language and environment for statistical computing. R Foundation for Statistical Computing, Vienna, Austria. https://www.R-project.org/

Rautio, P., A.-P. Huhta, S. Piippo, J. Tuomi, T. Juenger, M. Saari, and J. Aspi. 2005. Overcompensation and adaptive plasticity of apical dominance in Erysimum strictum (Brassicaceae) in response to simulated browsing and resource availability. Oikos 111:179-191.

Robel, R. J., J. N. Briggs, A. D. Dayton, and L. C. Hulbert. 1970. Relationships between visual obstruction measurements and weight of grassland vegetation. Journal of Range Management 23:295-297.

Rosentahl, J. P., and P. M. Kotanen. 1994. Terrestrial plant tolerance to herbivory. Trends in Ecology and Evolution 9:145-148.

Sachs, T. 1999. Node counting, an internal control of balanced vegetative and reproductive development. Plant, Cell, and Environment 22:757-766.

Simons, J. L., C. A. Napoli, B. J. Janssen, K. M. Plummer, and K. C. Snowden. 2007. Analysis of the decreased apical dominance genes of Petunia in the control of axillary branching. Plant Physiology 143:697-706.

Strauss, S. Y., and A. A. Agrawal. 1999. The ecology and evolution of plant tolerance to herbivory. Trends in Ecology and Evolution 14:179-185.

Tuomi, J., P. Nilsson, and M. Åström. 1994. Plant compensatory responses: bud dormancy as an adaptation to herbivory. Ecology 75:1429-1436.

Vail, S. G. 1992. Selection for overcompensatory plant responses to herbivory: a mechanism for the evolution of plant-herbivore mutualism. American Naturalist 139:1-8.

van der Meijden, E. 1990. Herbivory as a trigger for growth. Functional Ecology 4:597-598.

Westbury, D. B. 2004. Rhinanthus minor L. Biological flora of the British Isles. Journal of Ecology 92:906-927.

Wise, M. J., and W. G. Abrahamson. 2005. Beyond the compensatory continuum: environmental resource levels and plant tolerance of herbivory. Oikos 109:417-428.

\section{SUPPORTING INFORMATION}

Additional supporting information may be found in the online version of this article at http://onlinelibrary.wiley.com/doi/ 10.1002/ecy.2101/suppinfo 